\title{
PRACTICAL REASON NOT AS SUCH
}

\author{
Kenneth Walden
}

$\longrightarrow$

ONSTRUCTIVISTS THINK that value is a "construction" of the attitude of valuing. For a thing to be valuable, "is for that thing's value to be entailed from within the point of view of a creature who is already valuing things." I think this is a compelling conception of value. But it is also a bleary view. For it is consistent with many different notions about which value claims are correct, how they are to be constructed from an agent's practical point of view, and what constitutes such a point of view.

One front for these questions is the debate between Kantian and Humean constructivists. These philosophers disagree about whether we can discern anything substantive about the construction of value by looking at the activity of practical reasoning "as such.” A Kantian like Christine Korsgaard thinks we can. Using practical reason commits us to the Categorical Imperative, and from this we can derive specific duties and prohibitions. These normative claims can therefore be constructed from every practical point of view. ${ }^{2}$ In contrast, a Humean constructivist like Sharon Street denies that practical reason as such commits reasoners to much of anything - that no "substantive moral conclusions are entailed from within the standpoint of normative judgment as such." Instead, "the substantive content of a given agent's reasons," Street says, "is a function of his or her particular, contingently given, evaluative starting points." 3 So if you do not give a damn about pain on Tuesday or the suffering of others, and you can coherently and self-consciously maintain that indifference, then there is no ground for saying that you nonetheless have a reason to avoid that pain or ease that suffering.

This dispute acknowledges two potential grounds for normative correctness

1 Street, "What Is Constructivism in Ethics and Metaethics?" 367.

2 Korsgaard, Sources of Normativity. Another version of the view is defended by Markovits, Moral Reason, 145-62.

3 Street, "What Is Constructivism in Ethics and Metaethics?" 370. Defenses of views that could be reasonably called Humean include Street, "Constructivism about Reasons," and Lenman, "Humean Constructivism in Moral Theory." 
within the set of factors that shape a person's behavior. First there is "practical reason as such." This is the universal and perfectly generic faculty for practical deliberation tout court and so a factor in nearly every action performed by a rational agent. (The notion seems to be a descendant of Kant's "pure" practical reason: our capacity for reasoning practically abstracted from any empirical and so contingent conditions.) And then there are what Street calls our "particular, contingently given" evaluative attitudes-my abhorrence of Torquemada's cruelty, my admiration for Pollini's phrasing, my preference for Darjeeling in the afternoon.

But surely there are elements of our moral psychology that do not fit neatly into these pigeonholes - things that are neither particular evaluative attitudes nor features of practical reason as such. For brevity's sake I call these "further factors." I worry that neglect of these features' role in shaping what is entailed from our practical point of view can keep constructivism from being as sophisticated and catholic a view as it might be.

This worry prompts the questions I take up here: Might there be constructivist arguments in support of distinctive normative judgments whose starting point is these further factors? And might such arguments constitute a constructivist program that complements those advocated by Street or Korsgaard? Bigger questions are at stake as well, ones I am sneaking up on by entering into this intramural dispute between constructivists. They are questions about practical reason not as such. Are there contingent features of agents that are nonetheless best understood as genuine facets of their faculty of practical reasoning? And, if there are such features, might they play a role in determining which normative judgments are correct for an agent in something like the way that practical reason "as such" is supposed to? The debate between different flavors of constructivism is a good stalking horse for these questions because constructivists agree on a tight connection between practical reason and normativity.

The goal of this article is to defend an affirmative answer to all of these questions. The first step in doing this is identifying what I call "further factors" and saying why we should believe in them. This I do in section 1 . The second step is showing that they have some significance for which normative judgments are correct. I do this within the general setting of constructivism in section 2. In section 3 I suggest that this version of the view can contribute to the cause of establishing the extensional adequacy of constructivism. In section 4 I close with a brief discussion of the import of the notion of practical reason not as such. 


\section{FURTHER FACTORS IN THE PRODUCTION OF ACTION}

I said that there are features of our moral psychology that contribute to the actions we perform but fall in between the two poles that Street's and Korsgaard's dispute centers on-between practical reason as such and our particular evaluative attitudes. In this section I present two examples.

My first example is an item from the social scientist's toolkit. Someone insults Igor, and Igor challenges him to a duel. Why does Igor do this? One part of our explanation cites Igor's evaluative attitudes and beliefs. He has some end, and he believes that challenging his antagonist is a means to that end. But suppose Igor's challenge is part of a larger pattern in Igor's community: a constellation of interconnected practices and symbols centering around honor that are inculcated early in life and have a pervasive influence on the way of life is carried out by those in that community. In this case it would seem a full explanation of Igor's action must say something more. It must say something about this regularity and Igor's role within it.

Now suppose that when Igor arrives at the museum he patiently waits in line to purchase a ticket. Why does Igor do this? Here, too, one part of the explanation cites Igor's evaluative attitudes and beliefs. But this also seems incomplete. For one, Igor's behavior instantiates a distinctive regularity. If he is like most of us, his queuing is not the result of any weighing of ends and calculation about how to achieve them. Rather, his initial recognition of the row of idle people is as a queue-as a thing-to-be-waited-in. As such he never seriously entertains the possibility of striding past it; this possibility is "silenced" in his deliberations. ${ }^{4}$ The practical problem Igor faces is framed in a particular way: not how to get into the museum most quickly, but how to enter the queue. There are exigencies that might spur Igor to skip the line, of course, but these exceptions prove the rule. Skipping the line would be "cutting," i.e., a violation of the norm.

The obvious way to supplement our initial explanation of Igor's challenge is to say that Igor lives in an honor culture, that he has internalized a particular role in that culture, and that his challenge is prescribed by that role. Likewise, the obvious way to supplement our explanation of Igor's waiting in line is by saying that he has internalized a social norm, the norm of queuing, and that he is following that norm. When giving this sort of explanation the thing we are attributing to Igor is a psychological schema. As Sally Haslanger explains:

A schema consists in clusters of culturally shared concepts, beliefs, and

4 For a defense of the claim that social norms silence in this way, see Hlobil, "Social Norms and Unthinkable Options." 
other attitudes that enable us to interpret and organize information and coordinate action, thought, and affect. Schemas are public-think of them as social meanings conventionally associated with things in our social world, including language — but are also internalized and guide behavior. ${ }^{5}$

Our two examples of schemata differ along a few dimensions. The schema that Igor has internalized insofar as he is a "man of honor" is diffuse but pervasive: it informs many aspects of his life, but does so in relatively subtle ways. The queuing norm he has internalized, by contrast, is relatively localized in its application but highly specific in its guidance. They are, nonetheless, examples of the same basic phenomenon: the psychological internalization of some aspect of a social structure by an agent that directs her behavior in the ways necessary to conform to and reproduce that structure.

The action-guiding power of a schema is unique in a few respects. First, a significant part of its influence on our action consists not in directing our behavior in particular directions, as is characteristic of aims, ends, and projects, but by structuring what Haslanger calls a "choice architecture." Schemata "structure the possibility space for agency" by silencing options, making others salient, and "providing templates of interaction that favor (or discourage) certain forms coordination with respect to a resource, e.g., share, hoard, distribute; and by canalizing our attitudes accordingly." ${ }^{6}$ For example, an honor schema may silence overly conciliatory or artful ways of acting while making forceful and candid options more salient. It may offer templates for interaction between men and women whose employment allows both to maintain their status as honorable. And it may codify relatively well-structured rituals like the duel. This way of controlling behavior allows schemata to, as Pierre Bourdieu says, "generate and organize practices and representations that can be objectively adapted to their outcomes without presupposing a conscious aiming at ends."

Second, schemata direct behavior, in part, by penetrating an agent's perceptual and cognitive systems. As the anthropologist Paul Friedrich says about honor in particular, "[it] is a code for both interpretation and action; in other words with both cognitive and pragmatic components.... Honor consists of a system of symbols, values, and definitions in terms of which phenomena are conceptualized and interpreted." ${ }^{8}$ Thus, people who have internalized honor schemata

5 Haslanger, "What Is a (Social) Structural Explanation?" 126.

6 Haslanger, "What Is a (Social) Structural Explanation?” 128.

7 Bourdieu, The Logic of Practice, 53.

8 Friedrich, "Sanity and the Myth of Honor," 284-85. 
come to see particular acts as cowardly, attend to sleights of etiquette or deference, and instinctually associate masculinity with virtue in ways not typical of those lacking that schema.

Third, schemata are relatively resistant to change and updates-more so than, e.g., an agent's desires or aims. Changing or dislodging a psychological schema generally requires not merely changing one's mind about some judgment of fact or value, but retraining oneself in the modes of thought that the schema directs. As a result, there are notable examples of social norms and their attendant schemata persisting despite active and conscious resistance. ${ }^{9}$

Fourth, the guidance offered by schemata cannot be understood individualistically. That is, we cannot understand the way that the internalization of the queuing norm guides Igor's behavior without understanding that what he has internalized is something essentially public, as an instance of a norm that has been internalized by multiple agents for the coordination of their behavior. If Igor is the only person who queues (or ever has), then he is not acting out an internalized norm, but exercising a private caprice. Furthermore, schemata generally depend on publicly available material resources. The practice of queuing is a way to coordinate access to a scarce resource-a bank teller's attention, a ride on Space Mountain - and in turn uses the spatial and temporal resources that facilitate individual instances of queuing. This trade-off of resources must be equilibrated for the practice to be sustained. The explanatory use of psychological schemata brings these elements in train. If we say that Igor issued a challenge because he has internalized an honor schema, we are not merely locating a cause of his action within his own psychology (as we might understand an explanation in terms of beliefs and desires), but orienting Igor within a shared social practice.

If we want to cite schemata like these as examples of "further factors" in action, we face two questions. First, why should we countenance such things in the first place? The answer to this is that doing so gives us the best, most complete explanations of human behavior. In particular, relying on schemata offers a kind of explanatory power not found elsewhere. As Haslanger puts it, schemata "offer insight into why the particular individual behaved as he/she did, but [they] also contribute to our understanding of the individual as the instance of a type-a type defined by the conditions for existing at that node. By carving the explanadum across a broader range of possibilities (as a type, not a token), we can achieve better, more stable, explanations." 10

The second question is harder. How do schemata stand relative to the contrast described above? Can they be reduced to some combination of evaluative

9 See Bicchieri and Fukui, “The Great Illusion.”

10 Haslanger, "What Is a (Social) Structural Explanation?" 128. 
attitudes and the activity of practical reasoning as such? Or are they an example of what I call further factors?

The first reductive possibility is easily rejected: schemata are not features of practical reasoning as such since they are clearly contingent factors in action. At first blush it seems equally obvious that they cannot be assimilated to evaluative attitudes either. Our paradigms of valuing are attitudes such as liking, appreciating, admiring, preferring, wanting, hating, fearing, and scorning. It is hard to see how the psychological work of, say, an honor schema could be reduced to one or even a set of these.

Now, it is true that schemata often involve evaluative attitudes: valuing one's honor is arguably a constitutive component of internalizing the honor schema, and one may be motivated to internalize a norm in order to avoid censure or to gain the advantages of coordination that norms provide. And it is also true that some valuing attitudes can display some of the features of schemata I listed a moment ago. Love may be capable of structuring our choice architecture by making certain actions "volitional necessities." Desires can be "backgrounded." Attitudes whose objects are social, like patriotism, may be unintelligible outside of a social context. ${ }^{11}$ Nonetheless, it is hard to imagine fully reducing a schema to any array of desires, lovings, admirings, or fearings. Igor's internalization of the queuing norm is not just a matter of his valuing the coordination afforded by such norms, disvaluing the censure that awaits violators, valuing his self-conception as a rule-follower, or even some combination of these. We can imagine someone who has internalized the norm despite lacking these evaluative attitudes and someone who has failed to do so despite having them all. And even if we could produce a set of paradigmatically evaluative attitudes that collectively captured all of Igor's behavior that we associate with the schema, it is questionable whether this set would offer the same explanatory advantages as the schema itself. We may be able to identify the efficient cause of a particular action with this set, but it is not clear how it would afford us the structural understandingunderstanding Igor's act as a token of a larger regularity—that citing the schema does.

Upon initial inspection, then, psychological schemata look like bona fide examples of further factors. This initial impression may be too hasty, though. Street emphasizes that she has a technical notion of valuing in mind that is more liberal and structurally articulated than the standard conceptions. She says this while explaining that valuing is a very different attitude from mere desire. Much of the resistance to attitude-dependence theories of value, she thinks, can be traced

11 See Frankfurt, "Autonomy, Necessity, and Love"; Pettit and Smith, "Backgrounding Desire"; and MacIntyre, Is Patriotism a Virtue? 
back to the unfortunate assumption that relevant dependence basis is desire. This leads Street to recommend the attitude of valuing as a basis for her constructivism, and, more importantly, to suggest ways in which her notion of valuing is more inclusive and structurally complex than the ordinary notion of desire. It is possible that the assimilation of schemata to evaluative attitudes will look more promising once we understand this conception.

Street distinguishes valuing from mere desire in a few ways, but only one of them is relevant to our question. The attitude of valuing, she says,

is characterized by greater structural complexity than the attitude of mere desiring. We tend to think of "desiring" as directed at a single object or state of affairs: I desire a donut, for example, or to be rich or to be liked. Evaluative experience of the kind that confers value if anything does, however, is structurally a great deal more complicated than that. It often involves experiencing very specific features of the world as "calling for" or "demanding" or "counting in favor of" other very specific things. For example, I experience the fact that a friend lent me her car two months ago as counting in favor of saying "yes" to the favor she's asking me now; I experience someone's youth and inexperience as ruling out a harsh reply; and so on. Such states of mind are very different from simply wanting a donut... The attitude of valuing involves much more complex attitudes toward the world and one's own potential responses to it. ${ }^{12}$

This clarification is crucial, since a schema could simply be one of the "much more complex attitudes" that valuing issues in. Trouble is, Street does not say what these attitudes are, only what they are not necessarily. This makes it possible to read Street's characterization of the attitude of "valuing" as infinitely capacious - as potentially encompassing nearly every factor in an agent's behavior except the demands of practical reason as such. If we did this, then schemata would count as evaluative attitudes, but only trivially. ${ }^{13}$

Of course, how Street intends to use the word "value" is only of secondary importance. The real question concerns the "joints" of moral psychology: the theoretically important differences among the factors contributing to an agent's

12 Street, "Coming to Terms with Contingency," $43-44$.

13 Bernard Williams's characterization of the "subjective motivational set" is similarly open-ended: "It can contain such things as dispositions of evaluation, patterns of emotional reaction, personal loyalties, and various projects, as they may be abstractly called, embodying commitments of the agent" ("Internal and External Reasons," 105). The items on this list are so heterogeneous as to make me think that Williams means to include any item that is "subjective" and has the potential to explain behavior. In that case I do not see any reason to think these items comprise a kind. 
actions. Street is concerned with one such difference, between necessary and contingent factors. But this may not be the only or even the most significant division. So we should ask: Is there a theoretically significant difference in the way that our paradigms of evaluative attitudes_liking, admiring, fearing —influence an agent's behavior in the way that psychological schemata do?

I think there is, and the best way to describe it is through an analogy suggested by Cristina Bicchieri:

Like a collection of linguistic rules that are implicit in a language and define it, social norms are implicit in the operations of a society and make it what it is. Like a grammar, a system of norms specifies what is acceptable and what is not in a social group. And analogously to a grammar, a system of norms is not the product of human design and planning. ${ }^{14}$

Let us flesh out this analogy a little bit by thinking about factors at work in a person's linguistic behavior. First, there are the speaker's communicative aims: asserting that water is clear, offering a model of canine speciation, promising to help you move. These aims are highly contingent and up to the speakers to adopt. Then there are the conditions of language as such: publicity, recursivity, etc. In between these two poles lie such things as the grammars of individual languages. That English has a particular grammar is a contingent thing, but it is contingent a very different way from Igor's aim of using language to ask Mrs. Igor for a cup of tea. Likewise, speakers of a language have some control over their grammarthe grammar is what it is because of linguistic practices enacted by linguistic actors, and grammar can change over time-but this control is deeply attenuated when compared to the control they have over their own linguistic aims.

The internalization of a grammar shapes linguistic behavior in much the same way that psychological schemata shape behavior more generally. Grammar guides our linguistic behavior by structuring our "choice architecture": not by directing us to perform particular speech acts, but by giving us templates for formatting those speech acts. It penetrates our perceptual and cognitive systems: I need not consult a rule to recognize the problem with subject/verb disagreement-it just sounds wrong. It is sticky: I cannot simply decide to change the grammatical rules that I follow, even if they can slowly evolve over time. And it is essentially social: it cannot be understood independently of its connection to a public language spoken by other people with whom I want to communicate.

I go to the trouble of describing these parallels because I think it is obvious that there is a significant theoretical difference-a joint-in how the internalization of a grammar contributes to an agent's linguistic behavior and how 14 Bicchieri, The Grammar of Society, xi. 
that agent's contingent linguistic aims do the same. (Even though they are both, strictly speaking, contingent features of language use.) And so it would be a mistake to try to assimilate the former to the latter. There is an equally important and structurally analogous difference between psychological schemata and our paradigmatic evaluative attitudes. The distinctively grammar-like way that schemata guide agents' behavior sets them apart from our paradigms of valuing, and this, I suggest, makes them good examples of further factors.

My second example of a further factor involves an agent's physical characteristics. Igor wants a sip of tea, so he stretches out his arm, grasps his cup, retracts his elbow, and imbibes. Why does Igor do all these things? Because that is the way to get the tea, and it is the way to get the tea because Igor cannot lift the cup with his mind or magically teleport the liquid from cup to gullet. Call the complex of features that explain our actions in this way our embodiment.

We face the same two questions with this example as we did with our first. Why should we believe in such a quality? And is it a genuine example of a "further factor"? The answer to the first question is much the same as before. Explanations that adduce the fact that Igor is a creature with arms and fingers oriented in space without telekinetic powers afford us a structural understanding of Igor's actions insofar as they allow us to understand those actions as part of a greater regularity that encompasses other, similarly embodied agents. This explanatory benefit is a reason to believe in the quality. As for the second question, there should also be little temptation toward reducing embodiment to a set of evaluative attitudes. And since we can well imagine different sorts of creatures who are capable of practical reason but have very different physical constraints on their agency, we should not think that it reflects the demands of practical reason as such.

Instead, the main source of resistance to the suggestion that Igor's embodiment represents an interesting further factor in his acting is that it is not really a feature of Igor (qua agent) at all, but a nonnormative fact that Igor must reckon with while deliberating. Igor sipped his tea on Main Street instead of Elm Street because that is where the café is. So this geographical fact is one factor in Igor's behavior. But this is not a feature of Igor the way that his evaluative attitudes and capacity for practical reasoning are. So why think differently about his embodiment? The suggestion implicit in this question is to assimilate Igor's embodiment to the undistinguished swathe of nonnormative facts that he encounters in his deliberations. His having a body and all the capacities and restrictions that come along with that have the same significance for his behavior as geographical facts about which street has a café. And so they are not "further factors" in any interesting sense. 
Like the suggestion of assimilating schemata to the attitude of valuing, I think this proposal elides an important difference. We can get a handle on it by examining the phenomenology of choice. Suppose Igor is waiting for a flight and decides to have a cup of tea. To do this, he briefly considers all the shops near his gate, excludes those that do not sell tea, and heads off to the nearest one that does. By contrast, no one decides to have a sip of tea, entertains all logically possible ways of accomplishing that, and only then crosses off possibilities like teleportation that are incompatible with her embodiment. For agents embodied like us, the question of how to drink tea just is the question of how to bring one's body into the right kind of contact with tea, and not a special instance of a more general question that happens to apply to us.

One could reply that there are other potential explanations for this difference: the difference is explained by Igor's habituation to his body and that he could, in principle, become habituated to other things just as thoroughly. But the relationship between Igor's practical reasoning and his embodiment seems special in a few respects. For creatures like us, practical reasoning is in the first instance an embodied activity: a project that involves moving our bodies around in space and time. Our conception of practical reason as such - of a faculty for reflection and deliberation divorced from our embodiment-is an abstraction from this activity. This is a point Charles Taylor has emphasized. Our practical perspective on the world, he observes, "is essentially that of an embodied agent, engaged with or at grips with the world"-which is to say that "our perception as an experience is such that it could only be that of an embodied agent engaged with the world." ${ }^{15}$ For example, the world we face when we set about to do things is "oriented vertically, [where] some things are 'up, others are 'down'; and in depth some are 'near,' others 'far.' Some objects 'lie to hand,' others are 'out of reach': some constitute 'unsurmountable obstacles' to movement, others are 'easily displaced." 16 And when we face this world, we do so under certain constraints. We face it as "an agent who acts to maintain equilibrium upright, who can deal with things close up immediately, and has to move to get to things farther away, who can grasp certain kinds of things easily and others not, can remove certain obstacles and not others, can move to make a scene more perspicuous; and so on." ${ }^{17}$ Finally, our training in practical reasoning involves training in how to manipulate our bodies. The cultivation of a person's ability to recognize the abstract categories of means and ends begins with the infant's recognition that certain basic actions relieve certain forms of distress - that suckling relieves

15 Taylor, "The Validity of Transcendental Arguments," 23, emphasis added.

16 Taylor, "Lichtung or Lebensform," 62.

17 Taylor, "Lichtung or Lebensform," 62-63. 
hunger - and the capacity for intertemporal forms of reasoning, like planning, is rooted in the child's ability to tolerate the visceral discomfort that accompanies delayed gratification.

These features distinguish an agent's embodiment from the nonnormative facts she must deal with in her deliberations. We do not introduce embodiment as a special constraint on an antecedently abstract activity of practical reasoning, as we might introduce geographical facts about tea as constraints on instrumental reasoning toward the end of tea drinking. On the contrary, the more generic activity is secondary: it is an abstraction away from our usual way of going about practical reasoning, which is structured by our embodiment. This constitutes an important difference - a joint - between facts like a given café being on Main Street and those features of an agent that constitute her embodiment. In an important sense, then, an agent's embodiment is not exogenous to her practical reasoning: it conditions that reasoning, rather than acting on it as an external force or constraint. And for this reason I think we should accept that it is a genuine example of a "further factor."

In this section I have been trying to identify "further factors" in our actionthings that contribute to our behavior but cannot be reduced to the items that Humean and Kantian constructivists have focused on in their projects. I have offered two examples: the way a social structure is psychologically internalized by an agent through a schema and the way an agent is embodied. Despite the hypothetical resistance I have considered here, I do not think the existence of these factors should be very surprising. The hard part of my case comes next: establishing that these things make a difference to what reasons an agent has.

\section{THE NORMATIVE SIGNIFICANCE OF FURTHER FACTORS}

To make this argument, I work within the broadly constructivist framework I adumbrated in the introduction. The primal idea of metaethical constructivism is that the correctness of normative facts is a function of what can be constructed from an agent's practical point of view. This means that two things figure in normative correctness: the agent's practical point of view - the construction basisand the methods employed in performing the construction. For constructivists, then, the question of whether the further factors I identified in the previous section have normative significance is the question of whether they affect either of these components.

A prima facie case can be made for including further factors in either of them. Psychological schemata and forms of embodiment shape the way that agents view the world when engaged in practical thinking, and they do so in very deep 
and persistent ways, so it seems fair to say they are part of the agent's "practical point of view." On the other hand, these factors structure agents' practical reasoning in distinctive ways: they structure their choice architecture, organize their experience into discrete practical problems, and make particular solutions to those problems salient. So insofar as our construction procedure is supposed to reflect the work undertaken by practical reason, it seems likely that further factors will play a role here as well.

There are some reasons to be skeptical about both possibilities, however. First, one could object that, for the purposes of normative construction, an agent's "practical point of view" ought to include only those attitudes that reflect her full-fledged normative judgments - her evaluative attitudes. Further factors may condition an agent's practical outlook without earning her endorsement, but precisely because they lack such endorsement we ought not see them as part of the practical point of view from which normative correctness is constructed. Second, in determining normative correctness we want to know what is really entailed by these attitudes, not what agents might suppose is entailed by them given their idiosyncratic styles of reasoning. This means taking practical reason as such as our standard, not the parochial procedures employed by imperfect agents.

Those moved by these objections are likely to favor a more austere constructivism. Street's program is a good example. For her, the practical point of view is "the set of all of the relevant agent's normative judgments, minus the normative judgment whose correctness is in question." ${ }^{18}$ Normative correctness is constructed from this point of view by a particular conception of the demands of practical reason as such: by standards grounded in the constitutive nature of the attitude of valuing as such.

Just as it is constitutive of being a parent that one have a child, so it is constitutive of taking oneself to have conclusive reason to $Y$ that one also, when attending to the matter in full awareness, takes oneself to have reason to take what one recognizes to be the necessary means to $Y$. One cannot take oneself to have conclusive reason to $Y$ without taking oneself to have reason to take the means to $Y .^{19}$

These constitutive features lay down standards by which normative judgments can be deemed correct or mistaken. Thus, from the point of view of someone who takes himself to have a reason to $Z$, the judgment "I have a reason to $Y$," where $Y$ is the necessary means to $Z$, is correct, and the judgment " $I$ have no rea-

Street, "Constructivism about Reasons," 223-26. Here I follow Street's usage and treat "normative judgment" and "evaluative attitude" as equivalent.

Street, "Constructivism about Reasons," 228. 
son to $Y$ " is incorrect-just by dint of the constitutive standards implicit in the attitude of taking oneself to have a reason. Through the repeated application of these standards to an agent's evaluative attitudes, the entailments of that agent's practical point of view are constructed.

My argument for the normative significance of further factors is that more austere constructivists face certain problems precisely because they deny this significance. The prima facie case is easy enough to sketch, but it has to be filled out with examples. The standards that Street says are introduced by the constitutive nature of valuing include things like: if $A$ values $e$, and $m$ is a necessary means to $e$, then $A$ values $m$; if $A$ values $r$, and $r=s$, then $A$ values $s$; if $A$ values all $x$ that are $F$ and $c$ is $F$, then $A$ values $c .^{20}$ These are essentially the laws of first-order logic applied to the valuing operator plus one distinctively practical constraint, the instrumental principle. They are very undemanding standards, and this should make us worry that combining them with an agent's evaluative attitudes will not suffice to settle many of the questions about normative correctness that ought to be answerable.

Some examples will help bring this out. Suppose that Igor has internalized an honor schema and someone has insulted him. Does Igor have a reason to challenge this person to a duel? Intuitively, he does-or rather, that is what we expect someone who thinks that normative correctness is constructed out of an agent's practical point of view to say. But can we get this conclusion out of Street's constructivism? We may be tempted by the following argument:

1. Igor values his status as an honorable man.

2. Therefore Igor has a reason to maintain this status.

3. Issuing a challenge is the necessary means to preserve his status.

4. Therefore Igor has a reason to issue a challenge.

Here the conclusion is meant to follow from the constitutive standards of valuing in precisely the way Street describes. But there is a hitch: it is not clear that (3) is true. (Or even that Igor judges it to be true, though for Street what matters is the fact, not the agent's opinion.) For it may be possible for Igor to preserve his status by offering a trenchant diagnosis of what is wrong with the practice of dueling and convincing members of his community that honor is really about law and respect rather than vengeance. Or for Igor to preserve his status by finding some fiendishly clever way to humiliate the person who insulted him. Or for Igor to avoid losing face by failing to issue the challenge, but then doing something so stupendous that his honor is preserved all the same. These may be remote possibilities, but they serve to underscore the larger point. The condition on the

20 Street, "Constructivism about Reasons," 227-31. 
standard that Street says is constitutive of valuing — being a necessary meansis quite strong, and that makes the normative force of the standard weaker than we might have expected. ${ }^{21}$

This is an example of the underdetermination I think Street's view faces. There are a few ways she might reply. The first is to accept the underdetermination while denying that it is a defect. Street is open to this possibility in some cases. Sometimes, she says, "the standards legislated by a person's other normative judgments, coupled with all the relevant non-normative facts about necessary means, etc., are insufficient to yield a result one way or another."22 This is a sensible suggestion when a person is ambivalent or apathetic - when his evaluative attitudes produce a "tie" between different judgments or he simply lacks the relevant attitudes-but it seems inapt here. Igor is neither ambivalent nor apathetic in this situation. Rather, the apparent underdetermination seems to arise from the constitutive standards of Igor's evaluative attitudes and the nonnormative facts failing to mesh in a way that settles the case. Moreover, we should be reluctant to bite the bullet and accept underdetermination in this sort of case, since it has the potential to generalize the innumerable similar cases in which an agent's behavior is mediated by an internalized schema.

The second response would have us try to overcome the underdetermination with further evaluative attitudes. That is, we could attribute enough evaluative attitudes to Igor such that, when combined with a sufficiently fine-grained picture of the nonnormative facts, the question of Igor's reasons would, after all, be settled by even a very weak conception of scrutiny. For example, we could observe that Igor's alternatives to issuing a challenge may require him doing things he is reluctant to try or lead to outcomes that he disfavors. Of course, we would also have to assess the likelihood of success in each instance. If we amend premise (3) in this way, it would look like an expected utility calculation: for all $\varphi$ such that $\varphi$ is a logically possible alternative to issuing a challenge, the probability that

There is a complication here that I am bracketing. Street defines normative correctness in an unusual way. Instead of giving a constructive definition of normative correctness, she gives a constructive definition of normative mistakenness and defines correctness as, essentially, non-mistakenness. Thus for her a judgment is, so to speak, correct until proven mistaken. (We can see how unusual this is by comparing it to mathematical constructivism, in which the analogous view would have it that a mathematical proposition is correct unless a counterexample can be constructed.) One consequence of this definition is that the underdetermination I am alleging shows up in a unique way. Instead of entailing that there are $p$ for which neither $p$ nor $\sim p$ is correct, it entails that there are $p$ for which both $p$ and $\sim p$ are correct since neither judgment violates the weak standards set out by the constitutive requirements of the agent's evaluative judgments.

Street, "Constructivism about Reasons," 236. 
$\varphi$ succeeds in preserving Igor's honor times the degree that he values $\varphi$-ing and its consequences is less than the probability that issuing a challenge preserves his honor times the degree to which he values that option and its consequences. Or something along those lines.

Rejiggering premise (3) like this could render the argument sound, but it would mean attributing a multitude of relatively nuanced evaluative attitudes to Igor-ones about all the various $\varphi$-ings and their potential consequences. ${ }^{23}$ There are a few reasons not to take this path. First, it is not obvious why we are entitled to attribute such a wide range of subtly variegated evaluative attitudes to Igor. It is unlikely that he has consciously formed all of these attitudes, since many concern esoteric options. Rather, we probably feel justified in attributing them in a purely dispositional fashion. We need to attribute these attitudes, we might say, in order to explain Igor's behavior in a case exactly like the one we are thinking about. But this is not true. As we have seen, we can get a better explanation of Igor's behavior by saying that he has internalized a psychological schema associated with honor culture, and we can do so without attributing a bounty of evaluative attitudes. This explanation is better both because it gives us the structural insight into Igor's behavior that social explanations offer and because it is more psychologically parsimonious.

Second, this would mean understanding Igor's evaluative attitudes in a radically dispositional way - as consisting in anything we need to attribute to him in order to explain his behavior on a roughly Humean model of explanation. This seems to undercut the idea that was cited to motivate austere constructivism-namely that the practical point of view should be understood to include only those commitments genuinely endorsed, rather than factors that affect an agent's action without this kind of acceptance.

Finally, the proposal has the result that the construction of Igor's reason looks hairy, since it depends on something like a complex, expected value calculation, even though, for Igor, the existence of a reason to issue a challenge could not be more obvious. Of course, we should not insist that our construction procedure mimic an agent's actual reasoning, but in this case there is not anything to suggest that Igor's immediate recognition, without adverting to value calculations, represents an error in his reasoning.

For these reasons, I think we should be reluctant about overcoming the apparent indeterminacy this case reveals simply by adding as many additional evaluative attitudes as needed to produce a version of premise (3) that renders the argument sound.

23 Cf. Street, “Constructivism about Reasons," 233n42, in which she defends liberality about the attribution of evaluative attitudes. 
The final possibility is to suppose that there are additional constraints on the project of scrutiny-the construction of normative correctness-than Street imagines. These can be grounded in a more robust conception of practical reason as such, or in some further, contingent feature of Igor. The former is the sort of proposal we might associate with Kantian constructivists: practical reason as such involves more than Street's quasi-logical constraints, and these can help settle whether Igor has a reason to issue a challenge. In principle this strategy could work, but the actual proposals from Kantian constructivists seem poorly suited to the task. Korsgaard suggests that everyone is committed to valuing humanity as such. It is not clear how this particular value is going to help in Igor's case, except, perhaps, by saying that the whole business of dueling is irrational. Markovits says that reason requires us to achieve a higher degree of coherence than logical consistency - what Kant calls "systematic unity" in the first Critique. I am not sure how this would help either, since the problem does not seem to be that Igor's values are inadequately systematic, but that it is not clear what is entailed by a set of more or less systematic values.

That leaves one option for overcoming the underdetermination, that a contingent feature of Igor's moral psychology, beyond his evaluative attitudes, bridges the gap. Here we find an obvious proposal: our original argument that Igor has a reason to issue a challenge is sound-and so (3) is true-but only relative to a background framework partly constituted by the honor schema Igor has internalized. By this I do not mean that Igor believes that (3) is true. I mean that it is objectively true as a claim about the "choice architecture" he confronts when deliberating about how to respond to the insult: given the choices made salient by the honor schema, issuing a challenge is the necessary means. Insofar as Igor internalized this schema, the possibility of elaborate practical jokes or stirring speeches about the evils of retribution are not live options, so issuing a challenge really is the necessary means to maintaining Igor's honor. It is not logically, metaphysically, or nomologically necessary, of course. It is practically necessary in the sense defended by Williams: "what I recognize, when I conclude in deliberation that I cannot do a certain thing, is a certain incapacity of mine. I may be able to think of that course of action, but I cannot entertain it as a serious option." ${ }^{4}$ Thus we can construct Igor's reason to issue a challenge in a quite straightforward way if we understand the scrutiny of our evaluative attitudes-our construction procedure-as constrained by the same choice-structuring assumptions as Igor's own deliberations. This is the option I recommend.

The suggestion comes into better view with another example. Suppose Igor has internalized a queuing norm. One morning he finds himself wanting a cup 24 Williams, "Practical Necessity," 128. 
of tea on his way to an important appointment. He stops by a café and finds a line, one long enough that he will be late if he waits in it. Intuitively, the pro tanto reasons that Igor has arising from his valuing a cup of tea and getting to his appointment on time are in conflict, and so Igor needs to consider which value is more important to him. But how, exactly, does that conflict arise? The natural thought is that it is a nonnormative fact that Igor cannot both get tea and arrive at his appointment on time. But this is not strictly true. He could cut the line. Those queuing up are likely to be grumpy about it, but if he is insistent enough and offers elaborate justifications, he will probably prevail. So this is not enough to explain the alleged conflict. This appears to be another example of underdetermination.

One thought for overcoming it, akin to the option we considered above, would be to add that Igor values the coordination offered by the queuing norm or fears the reproach he would suffer for violating the norm. These attitudes would then give him reasons not to cut and so entail the conflict that we seem to find in his case. But it is not obvious that Igor needs to have these evaluative attitudes in order to face a conflict in this case. He could be skeptical about the value of the queuing norm. And he may be aloof enough that public reproach does not bother him. Even this version of Igor, it seems, faces a conflict between his competing reasons. If he has internalized the norm, then cutting is not an option, whether he likes the norm, is fearful of reproach, or not. He really cannot get tea and arrive on time. As in the previous example, our initial diagnosis of Igor's normative situation seems apt. Igor's evaluative attitudes really are in tension here, and this tension arises without mediation by auxiliary evaluative attitudes. But we can appreciate this tension only if we view Igor's practical point of view relative to certain background assumptions about the choices available to him-assumptions which, from Igor's point of view, are supplied by his internalization of the queuing norm.

In both of these cases I have suggested, first, that Street's constructivism cannot construct a reason that Igor seems, intuitively, to have and, second, that our intuitive way of understanding how Igor's reasons are constructed from his evaluative attitudes is correct, but only relative to a "background framework." These claims leave us with two questions. What are these "frameworks"? And what role, exactly, do they play in the construction of normative correctness?

I will not attempt a direct and comprehensive answer to these questions but instead approach them by way of analogy. The problem I have been saying Street's constructivism faces is similar to one confronted by philosophers of science. Scientific theories are underdetermined by the observations meant to support or refute them. For any set of observations, no matter how large, the class 
of theories compatible with them-where we understand compatibility by the standards of logic alone-will be infinitely large. Only those theories that are logically inconsistent with one or more observation sentences will be ruled out, and that leaves an infinite remainder. This means that if we want to know which theory-or small set of theories - is supported by a set of observations, we are going to have to rely on something more than the meager constraints of logic.

Many philosophers of science have argued that, in practice, scientists overcome this underdetermination by relying on certain contingent but relatively well-entrenched background structures that shape how inquiry proceeds within particular research programs. These structures-Kuhn gave them their most famous name, "paradigms"-play a distinctive role in theory construction. ${ }^{25}$ They define which sorts of problems are to be addressed in a given inquiry and sketch templates for solutions. They fix a representational scheme in which problems, observations, and explanations are to be couched. They precisify standards of theoretical coherence and articulate standard protocols for resolving violations of those standards, often by implicitly arranging theoretical goals into a hierarchy. They present experimental exemplars that guide empirical practice. They give operational glosses on vague theoretical values like simplicity and fecundity. This background work allows scientists to approach observations not as an undifferentiated heap that is compatible with an infinite number of theories, but as inputs into an articulate problem-solving apparatus.

If Kuhn's descriptive claim about the role of paradigms is correct, then it seems inevitable that paradigms will have a coordinate effect on epistemic normativity-on what the scientist's epistemic reasons are. For someone working within the paradigm of Skinnerian psychology, discovering some cognitive difference in a person is a reason to seek some corresponding conditioning mechanism in their environment and thus to design particular sorts of experiments crafted to discern such a mechanism. For someone working in a Newtonian paradigm, a certain style of solution to a mechanics problem will be epistemically appropriate-one assuming the laws of motion and done in the mathematicalized style of the Principia. For the phlogiston theorist, the cessation of combustion is a reason to think that the ambient air has been completely phlogisticated. This

25 Kuhn, The Structure of Scientific Revolutions. I use Kuhn as my example here because of his familiarity to most readers, but one can find devices that play similar roles in many places: the neo-Kantians' (Hans Reichenbach, Michael Friedman) notion of the "relative" or "contingent" a priori, Imre Lakatos's "research programs," Michel Foucault's "savoir," Ian Hacking's "historical" a priori, and Bas van Fraassen's "stances." There are important differences between these notions, but for my purposes they are not significant. See also Thompson, Life and Action, who emphasizes the role of life forms and practices in constituting normative standards, albeit by means rather different from those explored here. 
connection between paradigm and epistemic normativity seems inescapable if we acknowledge that scientists' actual methodology-at least when it meets some minimal standard of sophistication - can affect what epistemic reasons they have. Without this concession, it would be hard to avoid the conclusion that the Skinnerian, the Newtonian, and the phlogiston theorist were not only mistaken in their picture of the world, but fundamentally irrational. (All this is to say nothing about the thorny question of whether the choice between different paradigms is subject to the standards of rationality, much less about the truth of the central claims of Skinnerian psychology or phlogiston theory.)

Of course, the way a paradigm conditions the scientist's epistemic reasons will be different from the way that her observations do. When determining what epistemic reasons arise in virtue of these observations, we do so by assuming features of the paradigm as fixed background conditions on the epistemic endeavor to which the reasons attach. That is, when working out what epistemic reasons a scientist has, we assume that she is solving a certain kind of problem, that certain features are desired in a solution, that certain sorts of experimental apparatuses and protocols are called for, that phenomena are to be represented in particular ways, that certain techniques are appropriate, and that a certain basic picture of the world obtains. In this way, the constraints a paradigm places on the determination of the scientist's epistemic reasons parallel those that it places on the same scientist's actual methods.

The notion of a "background framework" I have in mind is a rough practical analogue of these paradigms, and the constructivism I am proposing accords them analogous normative significance. Like Kuhnian paradigms, these background frameworks are not grounded in the demands of reason as such, and one can in principle be abandoned in favor of another. They are nonetheless relatively stable fixtures of practical reasoning that perform several crucial tasks that help us overcome the underdetermination of normative correctness by evaluative attitudes. They define a practical problem space by structuring an agent's choice architecture. They fix a scheme in which both values and practical problems are represented. They lay down standards of coherence for evaluative attitudes (ones stronger than those imposed by the constitutive standards of valuing). They specify protocols for resolving incoherence, often by implicitly arranging values into a hierarchy. They offer exemplars of practical problem-solving that guide agents' deliberations. They give operational glosses on vague values like courage and justice. The constructivism I am proposing as a remedy to the underdetermination of Street's is distinguished by the claim that background frameworks so understood also constrain the construction of normative correctness, and are, for that reason, normatively significant. 
The crucial question here is: Constrained how? The idea must be to see the problem of what is normatively correct for an agent as structured in the same way as the problem the agent herself faces. For example, if a background framework structures an agent's choice architecture in a particular way, then, for the purposes of constructing what is normatively correct for her, we should take it as so structured. If it specifies particular prima facie evaluative tensions, then, for the purposes of normative construction, we should understand those values as standing in a prima facie tension. If it couches an agent's evaluative attitudes in particular concepts, our construction should do the same. If the framework privileges particular templates for solving practical problems, then our construction should privilege the same templates when deciding what reasons an agent has when facing such a problem.

I cannot offer a full delimiting of the range of constraints these frameworks may impose, but, as my examples suggest, I think these background frameworks very often are partly constituted by the further factors in the production of action that I identified in the previous section. And so those factors affect what is normatively correct for an agent by affecting their background structures of practical thought. For example, because Igor is embodied in a particular way, it is a feature of his background framework that the problem of seeing Manet's Olympia up close just is the problem of maneuvering his body into a particular region of space. As such, his wanting to see the painting entails, without the aid of auxiliary premises concerning his other evaluative attitudes and nonnormative facts, that he has a pro tanto reason to move his body in a suitable way. ${ }^{26}$ By contrast, it is false that, for Igor, the problem of seeing Olympia up close just is the problem of flying to Paris and purchasing a ticket to the Musée d'Orsayeven if this is, in fact, the best way to see the painting - and so his wanting to see the painting does not entail that he has a reason to do these things, at least not without auxiliary premises. Similarly, because Igor has internalized an honor schema, an insult usually entails, often without any consideration of his other values, a reason to respond with a challenge, and because he has internalized a queuing norm, long lines and short time constitute a prima facie normative conflict. These are just the simplest and most direct ways that further factors can influence what is normatively correct for an agent, but if the claims of anthropologists like Clifford Geertz and Richard Shweder are correct, and there are reason to occupy a particular region of space" is not an enthymeme. For a related argument, see Brandom, Making It Explicit. 
profound socio-psychological differences between cultures, then we are likely to find equally profound normative differences. ${ }^{27}$

Let me summarize the argument of this section. I began with a simple case that constructivists should acknowledge the normative significance of further factors in the production of action. I then noted two concerns with this idea: (i) the agent's "practical point of view" should only include those attitudes that reflect full-fledged normative judgment, and (ii) normative correctness is a matter of what is really entailed by these attitudes, not what an agent's parochial form of reasoning would say is entailed. These concerns naturally lead to a more austere constructivism, of which I took Street's view as an example. My reply, which has taken up most of this section, is that this spare constructivism faces a problem of normative underdetermination, one which can largely be attributed to its austerity. There are a few ways we can address this problem. One is to bite the bullet and concede the indeterminacy. The second is to be very liberal in attributing evaluative attitudes to agents. A third is to introduce a stronger conception of practical reason as such. None of these seem likely to succeed. The alternative I think is most plausible and most in the spirit of constructivism is to understand the construction of normative correctness from an agent's practical point of view as being guided not just by practical reason as such, but by a background framework that reflects contingent factors that color the agent's practical reasoning. Doing this gives those further factors an indirect but distinctive normative significance.

With this strategy we avoid concern (i) entirely, since we are not expanding our conception of the "practical point of view." As for (ii), I think we have good reason to reject it. It assumes that what is "really" entailed from a practical point of view is a matter of what is entailed by the universal, essential standards of practical reason as such (in Street's case, by the constitutive standards of valuing). But we should accept this assumption only if we think there is a single notion of practical entailment appropriate to all agents in all circumstances, and that this notion is up to the task of settling all the questions of normative correctness we expect to be settled. This is exactly what I think the underdetermination we have encountered gives us reason to doubt.

\section{CONSTRUCTING MORAL REASONS}

Constructivism is frequently criticized for failing to capture our considered judgments about normative correctness. The view produces "too few" reasons

27 I am thinking of Shweder's Thinking through Cultures, and Geertz's The Interpretation of Cultures. 
because we can imagine cases in which we are inclined to say that an agent has a reason to do something even though she lacks the evaluative attitudes that would seem to be necessary for a construction of that reason from her practical point of view. She may, for example, be wholly and completely indifferent to the plight of a person on fire or to her own life, and thus have no reason, by the constructivist's lights, to douse the flames or take her medicine. And it produces "too many" reasons because agents might possess eccentric evaluative attitudes that would ground reasons that no one could really have: torture the innocent for amusement, count blades of grass, or be indifferent to her own pain so long as it is suffered on a Tuesday.

No single strategy is going to be adequate to this variety of objections. Kantian constructivists may have some success in answering them by appealing to the possibility that practical reason as such may ground moral and prudential reasons. Others will insist that constructivism is correct about these cases, and we will realize this if we better appreciate the psychology of the eccentrics involved. ${ }^{28}$ There is a place for both strategies. What I want to suggest here is very limited: the constructivism I articulated in the previous section can contribute to this project of demonstrating the extensional adequacy of constructivism.

I focus on just one area: the ability of the constructivist to make good on our judgments about moral reasons. If an agent has the appropriate pro-moral evaluative attitudes - valuing the welfare of others, taking themselves to have a reason to respect rights, etc. - then we will be able to construct reasons for this agent to behave in morally appropriate ways. But some common opinions about moral reasons suggest that this is not enough. Many philosophers are attracted to moral rationalism, the view that everyone has moral reasons, whatever their evaluative attitudes, and that these reasons systematically override or exclude ones grounded in contingent interests. The Humean constructivist obviously cannot deliver on this. The Kantian constructivist can-sort of. If her arguments succeed, then everyone has an unconditional and overriding reason to follow whatever moral principle they have shown is legislated by practical reason itself, i.e., the Formula of Universal Law or Formula of Humanity. But the path from these principles to the reasons operative in particular cases is far from clear: Does my having an unconditional and overriding reason to act only on maxims I can will as universal laws entail that I have a similarly unconditional and overriding reason to leave a note when I dent your fender? Does my having an unconditional and overriding reason to treat humanity always as an end in itself and never as a mere means give me an overriding and unconditional reason to break one man's

28 E.g., Street, "In Defense of Future Tuesday Indifference," and Williams, "Internal Reasons and the Obscurity of Blame." 
arm if it will save fifty others from death? The answer is unclear. This leaves the constructivist, even in the best-case scenario, with the problem of showing that those agents who lack the appropriate evaluative attitudes have moral reasons in the many cases in which the application of the Categorical Imperative is unclear (or, of course, arguing that they do not). Here is where I think the constructivism I just outlined can help the cause.

The key point will be about the normative effects of schemata. Some schemata will be connected to practices that we regard as purely conventional: queuing, fashion, and etiquette. But others will guide us in activities that seem morally significant: ones involving property, promises, others' bodies, or the categories of virtue. What is it that distinguishes moral schemata, customs, and social structures from ones of mere custom? A popular thought, which I adopt here, is that moral aspects of a social order are distinguished by being (constitutively) subject to certain forms of reflective criticism that mere customs are not. For example, Kurt Baier understands a "moral order" as

a social order which raises certain critical questions about its mores and which tends to modify them in light of the answers it gives to them. These questions therefore function as the society's own tests of soundness, that is, tests of the belief that certain directives contained in its mores (and possibly in those of other societies as well), and purporting to be moral directives and so to pass a certain appropriate test, really do pass it. ${ }^{29}$

On this picture, there are two senses of morality: a particular social practice of policing and reforming other parts of the "social order," and the parts of the social order-rules, standards, norms - that purport to pass the distinctive tests imposed by this practice.

In an effective moral order, agents will be socialized so that they can successfully participate in that order, both by engaging in the critical activities of morality and by being guided by the results of that practice—by morality as their order finds it. And this means internalizing a schema that inclines them to behavior adhering to the prescriptions of that order's morality. It is easy enough to guess what this will entail. These agents will be inclined to represent situations with the import that morality prescribes: they will see an item as someone else's property, a speech act as a promise, a challenge as a test of fortitude. They will tend to interpret the actions of themselves and others according to the scheme imposed by morality: certain acts are thefts, promise breakings, acts of cowardice. They will have their choice architecture structured in a certain way: opportunities for theft may be systematically excluded from the range of options considered, 
whatever action constitutes keeping a promise may be given default status, those actions that would be particularly vicious are silenced. And within the options that are considered, the social costs of contra-moral actions will be made particularly salient. This is the kind of psychological work that a schema must do in order to maintain agents' reproduction of the social order.

On the conception of constructivism sketched in the previous section, this moralization of an agent's background frameworks for practical thought will have consequences for what is normatively correct for them. What, exactly, these are obviously depends on the details of a given moral order and how it is internalized by the agents who enact it. That said, some general examples can be speculated about. An agent will not have reasons to perform actions that are excluded from consideration by her background framework. So if opportunities for petty theft are generally excluded from the choice architecture of agents socialized in a moral order, these agents have no reason to steal in these cases. On the other hand, if an option is bestowed with default status by an agent's background framework-e.g., because it would constitute keeping a promise-then this option will be prima facie choiceworthy for the agent. Other effects will be more indirect. The use of a particular scheme of representation that reflects the categories morality deems important—representing acts as theft, murder, promise breaking, etc. - and the association of these categories with particular evaluative and deontic vocabulary_ "forbidden," "permissible," "generous," etc.—will obviously not guarantee that an agent always has most reason to do what morality prescribes. But it will ensure that the demands of morality lie within the domain of practical thought for such agents. For these agents, adhering to the prescriptions of morality will always be an option to be taken or rejected. This will guarantee that morality is within the realm of things that agents could have reasons for. This may not sound like much, but it distinguishes morality from other normative systems, like dead religions or strange aesthetic practices, that lack a foothold in an agent's practical point of view. Finally, the increased salience of rewards and costs associated with pro- and contra-moral behavior will tend, in the long run, to foster evaluative attitudes that will ground reasons of moral compliance.

My claim is that morality will be normative in these and allied senses for agents who have internalized the schemata distinctive of social orders that are also moral in Baier's sense. What does this get us? Certainly not the strong version of moral rationalism I described a moment ago. Even for agents who have internalized a moral order, it will not guarantee that they have reasons to do what that morality advises (much less decisive reason). Instead, I have suggested more attenuated forms of normative "significance" that under some condi- 
tions ground reasons for action. And even these limited effects will be fragile, since schemata are not deliberative straitjackets. An agent's internalization of a particular schema may silence the option of stealing my tea or wallet or make promise-keeping a deliberative default, but the right circumstances and a strong enough desire for tea or cash can cancel these effects. Indeed, professional burglars, faithless schemers, and full-fledged morality critics can condition themselves to neutralize the pro-moral effects of the common schema and in doing so remove these elements from their background framework of practical reasoning. And this is to say nothing of those who leave a given moral order altogether. The proposal falls short of full-blooded moral rationalism in another respect as well. The conception of morality that has normative significance for agents on this picture is tied to a particular social order. This is not to say that it is arbitrary, since it must pass the tests of validity that are constitutive of the difference between morality and mere custom. (Something that Baier discusses at length. ${ }^{30}$ ) But there is room for significant variation from one community to another, so we are not going to find reasons for any objective conception of morality.

Even with these concessions, I think the possibility outlined here has the potential to improve the fortunes of constructivists in trying to produce a satisfactory account of the moral reasons that agents have. First, by locating some of the normative significance of morality in agents' background framework of practical thought, we are able to explain why, in certain instances, the normative force of morality is unconditioned by agents' particular evaluative attitudes. Concomitantly, we can explain how morality can, sometimes, exclude or override potential reasons for contra-moral action, e.g., by excluding those options from the agent's choice architecture. In this respect it is an improvement on what the Humean constructivist can do. Second, by showing how the conceptual categories of a particular moral order gain entry into agents' practical thought, we are able to explain why agents have the reasons we intuitively judge them to have in concrete, contextualized cases - why they have no reason to commit some petty theft-and not just a reason to follow a highly abstract formulation of the moral law. In this respect, it is a useful supplement to extant versions of Kantian constructivism.

If constructivists are going to offer an account of normativity that comports with our considered judgments, they will have to fight on many fronts. They will have to show how more can be grounded in our evaluative attitudes than we might have thought. They may very well have to show that the use of practical reason as such commits us to a general moral principle. They will have to show that some of our initial judgments about reasons are unfounded. What I 
am claiming here is not that the liberal conception of constructivism sketched in the previous section is a panacea for these problems, but that it can play an important role in allowing constructivists to capture more of the nuance of the normative world.

\section{PRACTICAL REASON NOT AS SUCH}

In closing, I want to briefly suggest that my argument here can be understood as a case for there being such a thing as practical reason not as such —-for the legitimacy of talking about the ways in which practical reason, and not just its objects, can be shaped by the contingent features of an agent's situation and constitution.

There are two issues at stake in this suggestion. One is descriptive: Does positing such a thing as practical reason not as such help us explain human behavior? My argument in the first section is devoted to showing that it does. I argued that further factors shape an agent's practical outlook and structure her deliberations in ways that go well beyond our paradigms of valuing. In particular, they condition an agent's capacity for practical reasoning without being features of practical reasoning as such in a fashion analogous to the role of individual grammars in linguistic reasoning.

The second is normative: Does this faculty help us explain what reasons an agent has, what is good for her, and so on? In the second section I argued that, insofar as we have roughly constructivist metaethical scruples, it is appropriate to recognize these factors as making a distinctive contribution to normative correctness. I characterized this contribution as one of background conditions on the construction procedure, but we could just as well understand the construction of normative correctness as undertaken by a single capacity of practical reason that encompasses both this "background framework" and practical reasoning "as such." We could, that is, say that differences among agents in further factors actually carve out different styles or forms of practical reason-and different standards of correctness - just as the deep differences in scientific method reflect, in Ian Hacking's terms, different "styles" of theoretical reasoning. ${ }^{31}$

Of course, to make good on this project we would need much more precise criteria of individuation than I have provided here. Nonetheless, I think these results give us some initial reason to countenance talk of practical reason not as such, both as part of the project of explaining action and understanding the grounds of normative correctness. Indeed, unless we are keen on the question of practical reasoning's essential commitments, the division between practical 
reason "as such" and other features of agents' practical thinking can look highly artificial.

Humean and Kantian constructivists are interested in this question, since their principal disagreement is over whether there are any substantive judgments that are normatively correct for all possible rational creatures. To them, the constructivism I have defended here might look like no more than a modest expansion of the Humean program, since it has no pretensions to establishing the universality of substantive normative judgments. But this is not the only important question we can ask about the etiology of normative facts. It matters whether the reasons we have to adhere to some set of norms-be they a particular conception of morality, a religious creed, or a racist ideology-are rooted in widespread but nevertheless personal evaluative attitudes, or in contingent features of practical thought traceable to agents' socialization or embodiment. This in turn makes a difference to anyone taking up a critical perspective on these norms. Suppose a person comes to question the morality, religion, or racism she hitherto thought was normatively appropriate for her. How should she respond? If the normative correctness reflects her evaluative attitudes and no more, then she should reevaluate (which may sometimes require substantial effort). But if they are grounded in her internalization of a social schema, then a more thoroughgoing transformation is necessary. She must retrain herself in the use of practical reason to extirpate the suspect influences, and, in some cases, try to overturn whatever social structures introduce and sustain those influences. ${ }^{32}$

The general point is that it is often not enough to understand that a certain judgment is normatively correct for us, nor that it is contingently or necessarily so. We must also understand why it is correct, which aspects of our constitution and situation contribute to that correctness, and how they so contribute. Appreciating the normative significance of those features that constitute practical reason not as such is a first step in that direction. ${ }^{33}$

\author{
Dartmouth College \\ kenneth.e.walden@dartmouth.edu
}

32 On this distinction in the case of anti-racism in particular, see Shelby, "Racism, Moralism, and Social Criticism," and Haslanger, "Racism, Ideology, and Social Movements."

33 I am grateful to many people for comments and helpful discussion. The following list is just some of these people; I apologize for the inevitable omissions: Richard Holton, Julia Markovits, Alice Phillips Walden, David Plunkett, Paulina Sliwa, Sharon Street, an audience at the Madison Metaethics Workshop, and two anonymous referees. 


\section{REFERENCES}

Baier, Kurt. The Rational and the Moral Order. Chicago: Open Court, 1995. Bicchieri, Cristina. The Grammar of Society: The Nature and Dynamics of Social Norms. New York: Cambridge University Press, 2006.

Bicchieri, Cristina, and Yoshitaki Fukui. "The Great Illusion: Ignorance, Informational Cascades and the Persistence of Unpopular Norms.” Business Ethics Quarterly 9, no. 1 (January 1999): 127-55.

Bourdieu, Pierre. The Logic of Practice. Stanford: Stanford University Press, 1992. Brandom, Robert. Making It Explicit. Cambridge, MA: Harvard University Press, 1994.

Frankfurt, Harry. "Autonomy, Necessity, and Love." In Necessity, Volition, and Love, 129-41. New York: Cambridge University Press, 1998.

Friedrich, Paul. "Sanity and the Myth of Honor: The Problem of Achilles." Ethos 5, no. 3 (Fall 1973) 281-305.

Geertz, Clifford. The Interpretation of Cultures. New York: Basic Books, 1977.

Hacking, Ian. “Language, Truth, and Reason.” In Rationality and Relativism, edited by Martin Hollis and Steven Lukes, 48-66. Cambridge, MA: MIT Press, 1982.

Haslanger, Sally. "Racism, Ideology, and Social Movements." Res Philosophica 94, no. 1 (January 2017): 1-22.

. "What Is a (Social) Structural Explanation?” Philosophical Studies 173, no. 1 (January 2016): 113-30.

Hlobil, Ulf. “Social Norms and Unthinkable Options." Synthese 193, no. 8 (August 2016): 2519-37.

Korsgaard, Christine. Sources of Normativity. New York: Cambridge University Press, 1996.

Kuhn, Thomas S. The Structure of Scientific Revolutions. Chicago: University of Chicago Press, 1962.

Lenman, James. "Humean Constructivism in Moral Theory." In Oxford Studies in Metaethics, vol. 5, edited by Russ Shafer-Landau, 175-94. New York: Oxford University Press, 2010.

MacIntyre, Alasdair. Is Patriotism a Virtue? Lindley Lectures, vol. 23. Lawrence, Ks: University of Kansas, 1984.

Markovits, Julia. Moral Reason. New York: Oxford University Press, 2014. Pettit, Philip, and Michael Smith. "Backgrounding Desire." In Mind, Morality, and Explanation: Selected Collaborations, edited by Frank Jackson, Philip Pettit, and Michael Smith, 269-94. New York: Oxford University Press, 2004. 
Shelby, Tommie. "Racism, Moralism, and Social Criticism." DuBois Review 11, no. 1 (Spring 2014): 57-74.

Shweder, Richard. Thinking through Cultures. Cambridge, MA: Harvard University Press, 1991.

Street, Sharon. "Constructivism about Reasons." In Oxford Studies in Metaethics, vol. 3, edited by Russ Shafer-Landau, 207-46. New York: Oxford University Press, 2008.

- "In Defense of Future Tuesday Indifference: Ideally Coherent Eccentrics and the Contingency of What Matters." Philosophical Issues 19, no. 1 (October 2009): 273-98.

- "What Is Constructivism in Ethics and Metaethics?" Philosophy Compass 5, no. 5 (May 2010): 363-84.

Taylor, Charles. "Lichtung or Lebensform: Parallels between Heidegger and Wittgenstein." In Philosophical Arguments, 61-78. Cambridge, MA: Harvard University Press, 1995.

—. "The Validity of Transcendental Arguments." In Philosophical Arguments, 20-33. Cambridge, MA: Harvard University Press, 1995.

Thompson, Michael. Life and Action: Elementary Structures of Practice and Practical Thought. Cambridge, MA: Harvard University Press, 2008.

Williams, Bernard. "Internal and External Reasons." In Moral Luck, 101-13. New York: Cambridge University Press, 1981.

—. "Internal Reasons and the Obscurity of Blame." In Making Sense of Humanity and Other Philosophical Papers, 35-45. New York: Cambridge University Press, 1995.

_. "Practical Necessity." In Moral Luck, 124-31. New York: Cambridge University Press, 1981. 